\title{
Head nodding: An unusual presentation of congenital brain tumor
}

\author{
Kapil Bhalla ${ }^{1}$, Shuchi Mehra ${ }^{2}$, Ashish Gupta ${ }^{3}$, Sanjiv Nanda ${ }^{1}$ \\ From Departments of ${ }^{1}$ Paediatrics and ${ }^{2}$ Microbiology, Pt BD Sharma PGIMS, ${ }^{3}$ Trauma Centre, Pt BD Sharma PGIMS, Rohtak, Haryana, India
}

Correspondence to: Dr. Shuchi Mehra, Department of Microbiology, Pt BD Sharma PGIMS, Rohtak - 124 001, Haryana, India.

E-mail: drkapilbhalla@gmail.com

Received - 23 May 2019

Initial Review - 11 June 2019

Accepted - 26 June 2019

\begin{abstract}
Congenital brain tumors (CBTs) in neonates are defined as tumors presenting within 60 days after birth. Teratoma is the most common type of CBT. A 37-day-old baby was presented to pediatric emergency with complaints of difficulty in breathing, refusal of feed, and lethargy. The baby had continuous head nodding, drooling of saliva, and slight hypotonia of lower limbs which mother, then confirmed, was present since birth. There was a history of admission to another hospital with similar complaints at day 15 of life, where the child was discharged after 5 days of hospitalization with a diagnosis of nasal block. Magnetic resonance imaging brain showed a large mass located centrally suggestive of either pilocytic astrocytoma or medulloblastoma. The patient expired after 6 months in a private hospital. The present case report aims to focuse the importance of an early diagnosis based on the clinical presentation and imaging studies followed by timely intervention which is critical as the prognosis is very poor.
\end{abstract}

Key words: Brain, Child, Congenital, Head, Tumor

$\mathrm{C}$ ongenital brain tumors (CBTs) in neonates are defined as tumors presenting within 60 days after birth. CBTs represent only $0.5-1.9 \%$ of all pediatric brain tumors and are extremely rare $[1,2]$. CBTs are classified mainly on the basis of their histology. Neonatal tumors are generally congenital in origin. However, specific histological and clinical features are observed in brain tumors presenting during the 60 days after birth in comparison to tumors presenting in the first 2 years of life.

Most of the brain tumors in infants are gliomas $(63 \%)$ such as astrocytomas (36\%) [3,4], whereas most common CBT in fetuses and neonates is teratoma which accounts for approximately $30 \%$ of all CBTs in fetus and neonates. The initial point of formation of brain tumors during fetal and neonatal life may be driven normal cell differentiation, particularly germ cell tumors, frequently occurring before 32 weeks of gestation contain similar cells that present during the normal embryonic development [2]. The findings further support the hypothesis that these tumors are aberrant and residually derived during central nervous system formation.

Biopsy and histopathological examination are essential for a definitive diagnosis of a brain tumor but difficult in cases of fetuses, and hence, diagnosis should be done on the basis of imaging findings, clinical features, and the course of the disease [2]. In particular, teratomas are usually associated with calcifications, which may be important for diagnosis. The present aims to highlight the need and importance of timely diagnosis and timely intervention with respect to the insufficient prognosis of CBT.

\section{CASE REPORT}

A 37-day-old baby brought to the pediatric emergency with complaints of difficulty in breathing, refusal of feed, and lethargy. There was a past history of admission to another tertiary care center with similar complaints at day 15 of life, where the child was discharged after 5 days of hospitalization with a diagnosis of nasal block. On careful examination, the baby had continuous head nodding, drooling of saliva, and slight hypotonia of lower limbs which mother, then confirmed, was present since the birth of the child.

On general examination, the vitals were stable. No abnormality was detected on the chest and per abdomen examinations. Anterior fontanelle was at the normal level, and there were no signs of raised intracranial pressure. Reflexes, crying, and activity were decreased. Sucking was absent. Initially, the baby was diagnosed as a case of probable sepsis with underlying pneumonia. The baby was started on the appropriate treatment for late-onset sepsis (LOS); however, laboratory workup and radiological examination did not show any evidence of LOS. Blood investigations showed hemoglobin $=15.6 \%$ $\mathrm{g}$, total leukocyte count $=15,000 / \mathrm{mm}^{3}$, platelets $=350,000 / \mathrm{mm}^{3}$, and dimorphic picture on peripheral blood film. C-reactive protein was negative. The cerebrospinal fluid examination was normal, and blood culture was sterile. Chest X-ray, ultrasound abdomen, serum ammonia, and blood gas analysis were within normal range.

Magnetic resonance imaging (MRI) brain showed a large mass located centrally suggestive of either pilocytic astrocytoma or medulloblastoma (Fig. 1). The parents were explained the poor prognosis of the disease, but they refused for the surgery of the 


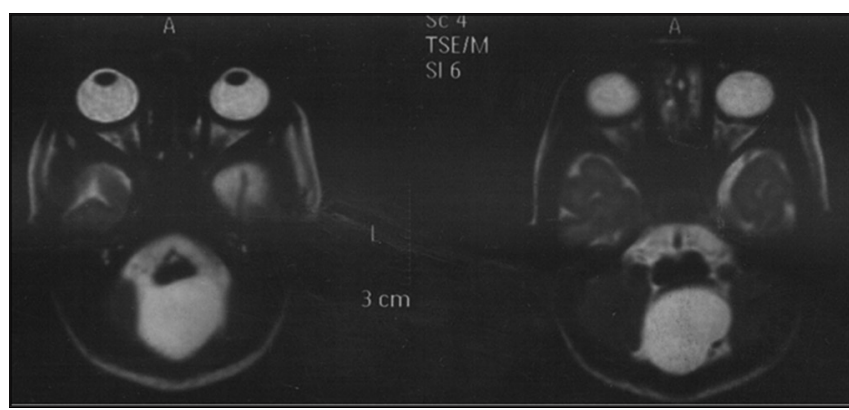

Figure 1: Magnetic resonance imaging brain showing a large mass located centrally suggestive of either pilocytic astrocytoma or medulloblastoma

patient and left the hospital against recommendation to continue treatment. Follow-up was done on the child, and it was found that the patient expired after 6 months in a private hospital.

\section{DISCUSSION}

Although rare, brain tumors represent one of the relatively larger groups of congenital neoplasias. Brain tumors can also be divided into two categories: intraparenchymal tumors and extraparenchymal tumors. Germ cell tumors belong to the former type. Intraparenchymal tumors invade the normal brain parenchyma and are malignant, except for certain intraparenchymal tumors such as choroid plexus papilloma, pilocytic astrocytoma, desmoplastic infantile astrocytoma, and craniopharyngioma. By contrast, extraparenchymal tumors such as lipoma and hemangioma are benign lesions [2].

CBT is arbitrarily divided into three categories: (i) definitely congenital, those presenting or producing symptoms at birth; (ii) probably congenital, those presenting or producing symptoms within the $1^{\text {st }}$ week; and (iii) possibly congenital, those presenting or producing symptoms within the first few months [1]. Hydrocephalus is the most frequent initial symptom reported in the literature [5]. Signs of increased intracranial pressure such as vomiting and papilledema are common presenting symptoms [6]. Clinical diagnosis is often difficult owing to nonspecific signs; some tumors are detected incidentally which happened in the present case. The diagnosis which can be prenatal or postnatal mainly depends on clinical findings and imaging studies.

On routine antenatal ultrasound examination, intrauterine hydrocephalus, enlarged biparietal diameter, or brain tumor is indicative of CBT [7]. It may be further confirmed by an MRI study of the fetus $[7,8]$. Other common presenting features are polyhydramnios, followed by respiratory distress and stillbirth [9]. Tumor markers such as alpha-fetoprotein and $\beta$-human chorionic gonadotropin may indicate malignancy pre-operatively. The prognosis is generally poor with a very low 1-year survival rate [6]. CBTs with benign histological features and compressing brain stem or pons generally have a poor prognosis. Choroid papillomas and astrocytomas usually have favorable outcomes $[10,11]$. It is difficult to predict the therapeutic effects and prognosis because of the challenges encountered in specifying the origin and type of tumor [2].

Gestational age of the patient may also provide useful information about the design of the diagnosis procedure.
Histological studies have indicated that before 22 weeks, tumor is often a teratoma or hamartoma. Germinal tumors can be observed between 22 and 32 weeks, whereas gliomas such as astrocytomas and glioblastomas can be detected after 32 weeks $[12,13]$. The several factors associated with the poor rate of prognosis in case of CBT are related to several factors such as malignant histological type, size and location of the tumor, immature stage of fetal development, and treatment-related complications [14].

\section{CONCLUSION}

The availability of the literature regarding the management of CBT is limited and is a major concern regarding any study concerned with CBT. The availability of the literature regarding the management of CBT is limited and is a major concern regarding any study concerned with CBT. Early diagnosis based on clinical presentation and imaging studies followed by timely intervention is very important as CBTs have a poor prognosis. however, more studies are required to establish guidelines for the management of CBT.

\section{REFERENCES}

1. Buetow PC, Smirniotopoulos JG, Done S. Congenital brain tumors: A review of 45 cases. AJNR Am J Neuroradiol 1990;11:793-9.

2. Sugimoto M, Kurishima C, Masutani S, Tamura M, Senzaki H. Congenital brain tumor within the first 2 months of life. Pediatr Neonatol 2015;56:369-75.

3. Rivera-Luna R, Medina-Sanson A, Leal-Leal C, Pantoja-Guillen F, ZapataTarre's M, Cardenas-Cardos R, . Brain tumors in children under 1 year of age: Emphasis on the relationship of prognostic factors. Childs Nerv Syst 2003;19:311-4

4. Jaing $\mathrm{TH}, \mathrm{Wu} \mathrm{CT}$, Chen $\mathrm{SH}$, Hung PC, Lin KL, Jung SM, Intracranial tumors in infants: A single institution experience of 22 patients. Childs Nerv Syst 2011;27:415-9.

5. Jellinger K, Sunder-Plassmann M. Connatal intracranial tumours. Neuropadiatrie 1973;4:46-63.

6. Maghrabi Y, Kurdi ME, Baeesa SS. Infratentorial immature teratoma of congenital origin can be associated with a 20-year survival outcome: A case report and review of literature. World J Surg Oncol 2019;17:22.

7. Ferreira O. Prenatal diagnosis of intracranial tumors: A case report. Pediatr Neurosurg 1993;19:84-8.

8. Hoff NR, Mackay IM. Prenatal ultrasound diagnosis of intracranial teratoma. J Clin Ultrasound 1980;8:247-9.

9. Takaku A, Kodama N, Ohara H, Hori S. Brain tumor in newborn babies. Childs Brain 1978;4:365-75.

10. Buetow PC, Smirniotopoulos JG, Done S. Congenital brain tumors: A review of 45 cases. AJR Am J Roentgenol 1990;155:587-93.

11. Isaacs H Jr. I. Perinatal brain tumors: A review of 250 cases. Pediatr Neurol 2002;27:249-61.

12. Kotulska K, Borkowska J, Mandera M, Roszkowski M, Jurkiewicz E, Grajkowska W, Congenital subependymal giant cell astrocytomas in patients with tuberous sclerosis complex. Childs Nerv Syst 2014;30:2037-42.

13. Laure-Kamionowska M, SzymanskaK, Biekiesinska-Figatowska M, GierowskaBogusz B, Michalak E, Klepacka T. Congenital glioblastoma coexisting with vascular developmental anomaly. Folia Neuropathol 2013;51:333-9.

14. Parmar HA, Pruthi S, Ibrahim M, Gandhi D. Imaging of congenital brain tumors. Semin Ultrasound CT MR 2011;32:578-89.

Funding: None; Conflict of Interest: None Stated.

How to cite this article: Bhalla $\mathrm{K}$, Mehra S, Gupta A, Nanda S. Head nodding: An unusual presentation of congenital brain tumor. Indian J Child Health. 2019; 6(7):392-393.

Doi: 10.32677/IJCH.2019.v06.i07.015 